\title{
Association of Glycated Hemoglobin and Dyslipidemia Inpatients with Type 2 Diabetes Mellitus in a K.M.C.H. Katihar, Bihar-A Retrospective, Cross-Sectional and Descriptive Study
}

\author{
Amresh Kumar' ${ }^{1}$ Jiut Ram Keshari², Manish Kumar', Manish Kumar ${ }^{4}$ \\ ${ }^{1}$ Assistant Professor, Dept. of Biochemistry, DMCH, Laheriasarai, Darbhanga, ${ }^{2}$ Additional Professor, Dept. of \\ Biochemistry, IGIMS, Patna, ${ }^{3}$ Associate Professor, Dept. of Pharmacology, IGIMS, Patna, ${ }^{4}$ Additional Professor, \\ Department of Physiology, IGIMS, Patna
}

\begin{abstract}
Introduction: Dyslipidemia is one of the major risk factors for cardiovascular disease in diabetes mellitusType2.The aim of the study was to understand the pattern of dyslipidemia and its association with glycated hemoglobin (HBA1C) among Type 2 diabetic.

Materials and Methods: This was a retrospective cross-sectional study to assess the relationship between glycemic control (as reflected by HBA1C) and serum lipid profile in Type2 diabetic patients which included a total of 200 Type 2 diabetic patients (104 males; 96 females; mean age years 62.91). Venous blood samples were collected from all the patients after at least $8 \mathrm{~h}$ fasting.

Results: HBA1C, fasting blood glucose (FBG), total cholesterol, triglycerides (TG), high-density lipoprotein (HDL) cholesterol, and low-density lipoprotein (LDL) cholesterol were analyzed. In male and female patient, serum levels of HBA1C, FBG, and LDL were not significantly different. As compared to males, female patients showed significantly higher serum cholesterol and HDL but significantly lower TG levels. Correlation between HBA1C and FBG was highly significant in this study. Both HBA1C and FBG exhibited direct correlations with cholesterol, TG, and LDL and inverse correlation with HDL; the magnitude of significance for all these lipid parameters being greater with HBAIC than FBG. There was a linear relationship between HBA1C and dyslipidemia. The levels of serum cholesterol and TG were significantly higher and of HDL significantly lower in patients with worse glycemic control as compared to patients with good glycemic control.
\end{abstract}

Conclusion: The findings of this study clearly showed that HBA1C is not only a useful biomarker of longterm glycemic control but also a good predictor of lipid profile.

Key words: Cholesterol, Diabetes mellitus, Dyslipidemia, Glycated hemoglobin, High-density lipoprotein cholesterol, Low-density lipoprotein cholesterol, Triglycerides

\section{Corresponding author}

\section{Dr. Manish Kumar}

Associate Professor, Department of Pharmacology, Indira Gandhi Institute of Medical Sciences,

Skeikhpura, Patna-800014, Bihar

Mob: 9304093698, Email: manu072@gmail.com

\section{Introduction}

Diabetes mellitus (DM) is cosmopolitan disease of the globe. DM is a group of metabolic disorders characterized by hyperglycemia either due to the lack of insulin secretion, or defects of insulin action or both. ${ }^{[1]}$ 
Recent reports showed that there were 171 million people in the world with diabetes in year 2000 and this is expected to increase to 366 million by $2030 .{ }^{[2]}$ It is associated with reduced life expectancy, significant morbidity due to specific diabetes related microvascular complications, increased risk of macrovascular complications (ischemic heart disease, stroke and peripheral vascular disease), and lessened quality of life.

Glycated hemoglobin (HbAlc) is usually used as a monitoring tool for measuring glycemic control in DM patients. It gives glycemic control status for last 120 days. ${ }^{[3]} \mathrm{HbA} 1 \mathrm{c}$ predicts risk for development of diabetic complication in diabetic patients. United Kingdom Prospective Diabetes Study (UKPDS) has revealed that risk of diabetic complications was strongly associated with previous hyperglycemia. Good glycemic control with decreased level of $\mathrm{HbA} 1 \mathrm{c}$ is likely to reduce risk of complications. ${ }^{[4]}$ Estimated risk of cardiovascular disease (CVD) has shown to be increased by $18 \%$ for each $1 \%$ increase in absolute $\mathrm{HbA} 1 \mathrm{c}$ value in diabetics. [5] The chronic hyperglycemia can damage several body organs due to microvascular and macrovascular complications. ${ }^{[6,7]}$

Macrovascular complications of diabetes include cardiovascular disease (CVD) such as stroke, which is the cause of death in $50 \%$ of diabetics. ${ }^{8,9} \mathrm{On}$ the other hand, microvascular complications of diabetes include diabetic nephropathy, neuropathy, and retinopathy. ${ }^{[10]}$

Cardiovascular risk of diabetes increases further if diabetes is related with dyslipidemia. However, this risk can be reduced by good management and control of both hyperglycemia and dyslipidemia. [11,12]

Dyslipidemia is one of the major risk factors for cardiovascular disease in diabetes mellitus Type2. ${ }^{[13-14]}$

It is estimated that currently India has 62.4 million people with diabetes mellitus. This is a major public health challenge, and it is increasing in epidemic proportions. Chronic hyperglycemia leads to micro-and macro-vascular complications. The lipid abnormalities in diabetics such as increased cholesterol, increased $\mathrm{LDH}$, high triglycerides (TG), and low high-density lipoprotein (HDL) are contributing to the mortality and morbidity. Worsening of glycemic control deteriorates lipid and lipoprotein abnormalities and particularly of diabetes mellitus. The combination of hyperglycemia, dyslipidemia, and hypertension produces enhanced atherogenic environment within the circulation. This leads to increased risk of ischemic heart disease, stroke, and myocardial infarction. Diabetes mellitus is considered as coronary heart disease equivalent. Insulin resistance, relative insulin deficiency, and obesity are associated with deranged lipid profile. The American Diabetes study has come to a conclusion that HBA $1 \mathrm{C}<7 \mathrm{mg} / \mathrm{dl}$ signifies optimal blood glucose levels. The management should focus on controlling diabetes and managing lipid levels which will reduce mortality and morbidity for ischemic heart disease and other diabetic complications. ${ }^{[14-22]}$

HBA1C is routinely measured to check the glycemic control over a preceding 8-12 weeks of time. It is used as an indicator for the state of glycemic control. Progression of the disease and the development of the complications in diabetic patients. The aim of the study was to examine the impact of the glycemic control on the lipid profile of Type 2 diabetic patients and to know the importance of HBA1C as an indirect indicator of dyslipidemia.

\section{Materials and Methods}

Study site- Department of Biochemistry and General Medicine, Katihar Medical College and Hospital, Katihar, Bihar.

Study duration-This study was carried out on diabetic patients during one-year period from October 2016 to September 2017.

Source of the data-History, physical examination, laboratory investigations were obtained from the medical records department.

Study design-Retrospective, cross-sectional and descriptive study

Method of collection of data-Total of 200 patient's records were accessed from the medical records department.

Inclusion Criteria: All diagnosed cases of Type 2 diabetes mellitus. 


\section{Exclusion Criteria:}

- Age below 18 years

- $\quad$ Type 1 diabetics

- Patients on lipid lowering agents

- Acute coronary syndrome
- $\quad$ Stroke

The lipid profile of the study was analyzed according to the ATPIII classification for identification of dyslipidemia, Low HDL $<40 \mathrm{mg} / \mathrm{dl}$. High low-density lipoprotein $(\mathrm{LDL})>190 \mathrm{mg} / \mathrm{dl}$, high cholesterol $>200$ $\mathrm{mg} / \mathrm{dl}$, and high TG $>200 \mathrm{mg} / \mathrm{dl}$.

\section{Results}

Table 1: Demographic data of diabetes mellitus (n-200)

\begin{tabular}{|l|l|}
\hline Male & $\mathbf{1 0 4}$ \\
\hline Female & 96 \\
\hline Mean age of year & 62.91 \\
\hline Age range (year) & $30-85$ \\
\hline
\end{tabular}

Table2: Lipid profile and HBA1C of diabetic patients

\begin{tabular}{|l|l|}
\hline Parameter & Mean \pm SD \\
\hline Total cholesterol & $149.73 \pm 47.37$ \\
\hline TG & $173.27 \pm 53.61$ \\
\hline LDL & $65.86 \pm 54.27$ \\
\hline HDL & $38.61 \pm 15.45$ \\
\hline HBA1C & $8.92 \pm 2.24$ \\
\hline
\end{tabular}

TG: Triglyceride, LDL: Low-density lipoprotein, HDL: High-density lipoprotein, HBA1C

Table3: Frequency of abnormal lipid profile status in all patients

\begin{tabular}{|l|l|l|l|}
\hline Dyslipidemia & Total & Male & Female \\
\hline Hypercholestrolemia & 72 & 34 & 38 \\
\hline Hypertriglyceridemia & 64 & 28 & 36 \\
\hline low HDL-C & 120 & 66 & 54 \\
\hline high LDL-C & 16 & 6 & 10 \\
\hline No abnormal lipid profile & 32 & 18 & 12 \\
\hline One abnormal lipid profile & 90 & 40 & 50 \\
\hline Two abnormal lipid profile & 56 & 28 & 28 \\
\hline$>$ Two abnormal lipid profile & 22 & 8 & 14 \\
\hline
\end{tabular}

LDL-C: Low-density lipoprotein cholesterol, HDL-C: High-density lipoprotein cholesterol 


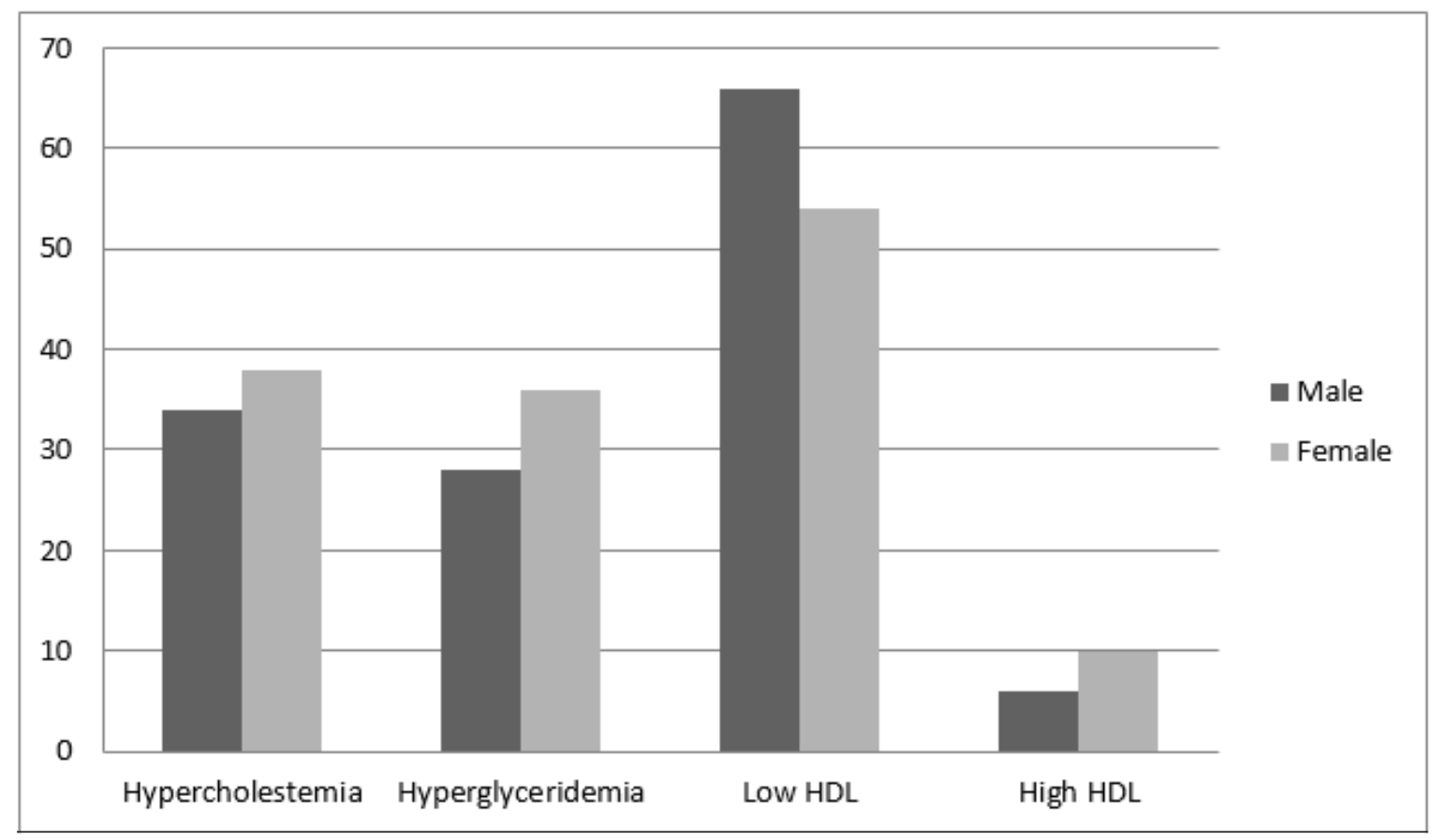

Figure 3: Lipid profile among diabetic patients

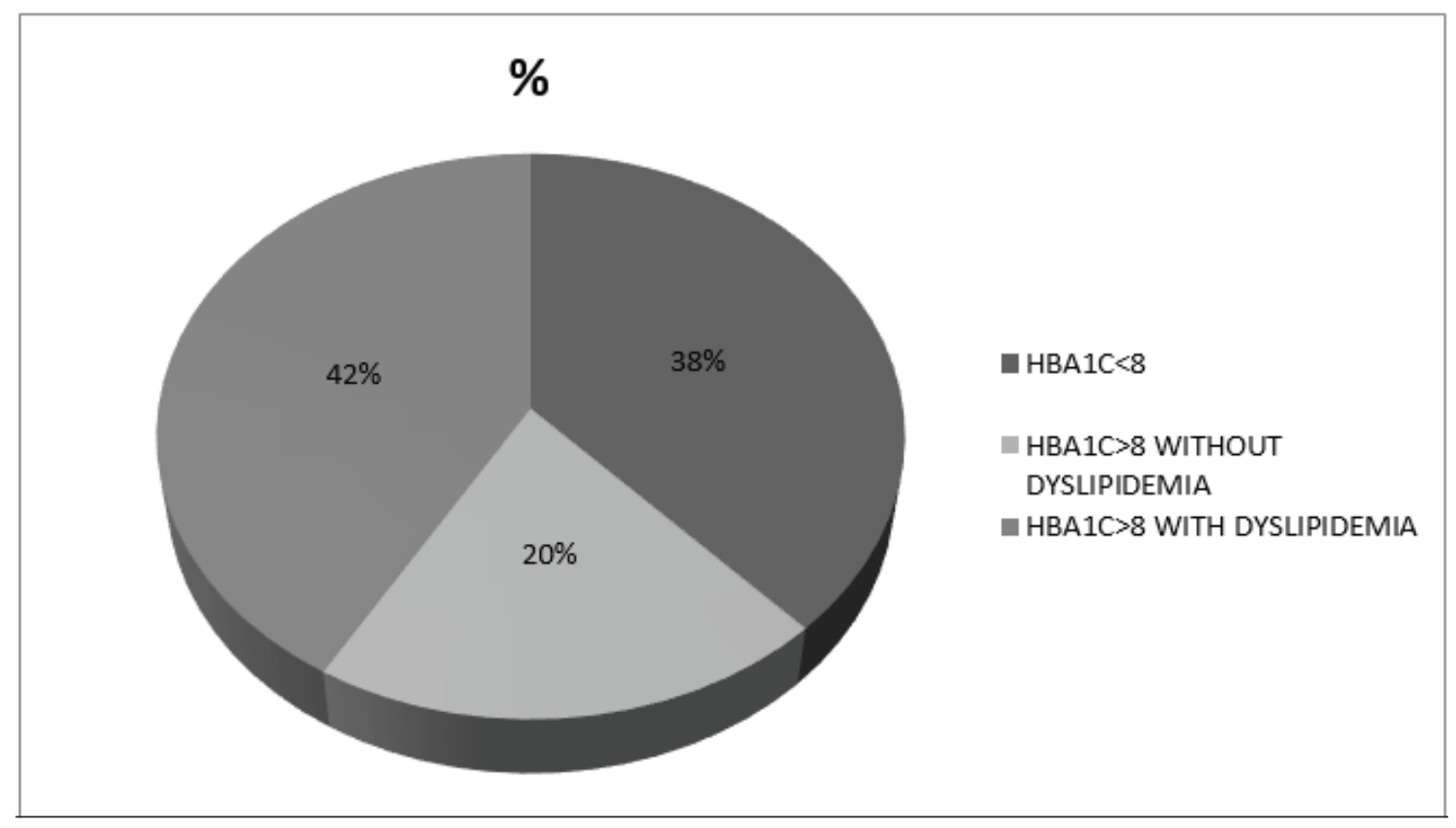

Figure 4: Correlation ship between glycated hemoglobin and dyslipidemia

A total of 200 patients with Type 2 diabetes mellitus were followed (104males and 96 females) (Figure1). The mean age was 62.91years with age range of 30-85years (Table1). Poor glycemic control (HBA1C $>8$ ) was seen in $124(62 \%)$ of total patients. Poor glycemic control was associated with dyslipidemia in $83(41.5 \%)$ of total patients, whereas $41(20.5 \%)$ accounted for poor glycemic control without dyslipidemia, the maximum frequency of abnormal lipid profile status in all patients was low LDL cholesterol (LDL-C) (Tables2 and3) and the age group with maximum patients with both dyslipidemia and higher HBA1C levels was 51-60 years. 


\section{Discussion}

This study was conducted on randomly selected 200 patients in a Govt. Medical College, Bettiah, Bihar, India. The lipid profile, fasting blood glucose (FBG), and HBA1C were investigated. This study reveals a high prevalence of hypercholesterolemia, hypertriglyceridemia, high LDL and low HDL levels which are well known risk factors for cardiovascular disease and incidence of poor glycemic control in Type 2 diabetic patients. Insulin affects the liver Apo-lipoprotein production. It regulates the enzymatic activity of lipoprotein lipase and cholesterol ester transfer protein. All these factors are likely cause of dyslipidemia in diabetes mellitus. ${ }^{[23]}$ Worse glycemic control with dyslipidemia was seen maximum in the individual of the age group 51-60 years (Figure 2). The core of this study revolved around identification of an association between dyslipidemia and poor glycemic control. The percentage of dyslipidemia individuals among the study population amounted to $62 \%$, among which $41.5 \%$ accounted for dyslipidemia with poor glycemic control (HBA1C $>8 \mathrm{mg} / \mathrm{dl}$ ), thus showing a positive correlation between dyslipidemia and HBA1C among patients in the population under study (Figure4). The pattern of dyslipidemia showed that $84 \%$ of the patients with abnormal lipid profiles and $16 \%$ of patients has no lipid profile abnormality; one lipid profile abnormality was seen in $45 \%$ of the study population, $28 \%$ had two lipid profile abnormalities, and $11 \%$ of the individuals had more than two abnormal lipid profile parameters. $84 \%$ among the study group of 100 patients had lipid profile abnormalities, among these 36 patients had hypercholesterolemia, 32\% had hypertriglyceridemia, $8 \%$ had high LDL-C, and 60\% had low HDL cholesterol levels (Figure3). Lipid abnormalities were more significant in women study patients in comparison with those of the male study patients (Figure3). The significant correlation between HBA1C and FBG is in accordance with various previous study done all over the world. Higher levels of FBG were noted in patients with poor glycemic control ( $84 \%$ of total study population of Type 2 diabetics). ${ }^{[23]}$

\section{Conclusion}

Association between lipid profile and HBA1C was evident in this study. Achieving the target in HBA1C will contribute in improving the lipid state, and hence may lessen the diabetic complications in Type 2 diabetic patients. Thus, by maintaining a good glycemic control, risk for the development of dyslipidemia and cardiac diseases can be reduced. Further studies should be conducted on a larger scale to significantly explore the role of $\mathrm{HbA} 1 \mathrm{c}$ in the development of dyslipidemia in type-2 Diabetic patients.

\section{Source of Support: Self \\ Conflict of Interest: Nil}

Ethical Clearance: Taken from K.M.C.H. Katihar, Bihar

\section{References}

1) Goldberg IJ. Diabetic dyslipidemia: statins versus fibrates in the treatment of diabetic dyslipidemia. Paper presented at: Symposium, program and abstracts of the 6st scientific sessions of the American Diabetic Association; 2001 June 22-26; Philadelphia, Pennsylvania

2) Wild S, Roglic G, Green A, Sicree R, King H. Global prevalence of diabetes estimates for the year 2000 and projections for 2030. Diabetes care. 2004;27(5):1047-53

3) Delamater AM. Clinical use of hemoglobin A1c to improve diabetes management. Clin Diabetes. 2006;24(1):6-8

4) Stratton IM, Adler AI, Neil HA, Matthews DR, Manley SE, Cull CA, etal. Association of glycaemia with macrovascular and microvascular complications of type 2 diabetes (UKPDS 35): prospective observational study. BMJ. 2000;321(7258):405-12

5) Selvin E, Marinopoulos S, Berkenblit G, Rami T, Brancati FL, Powe NR, Golden SH. Meta-analysis: glycosylated hemoglobin and cardiovascular disease in diabetes mellitus. Ann Intern Med. 2004;141(6):421-31

6) Genuth S, Alberti KG, Bennett P, Buse J, Defronzo R, Kahn R, et.al. Follow-up report on the diagnosis of diabetes mellitus. Diabetes Care. 2003;26(11):3160-67,

7) UK Prospective Diabetes Study (UKPDS); VIII Study design, progress and performance. Diabetol. 1991; 34(12):877-90 


\section{Sex Distribution}

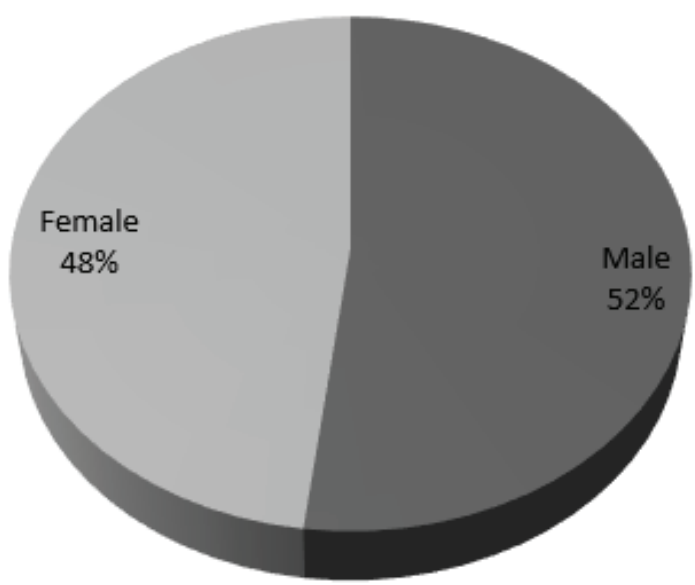

Figure 1: Sex distribution among study population

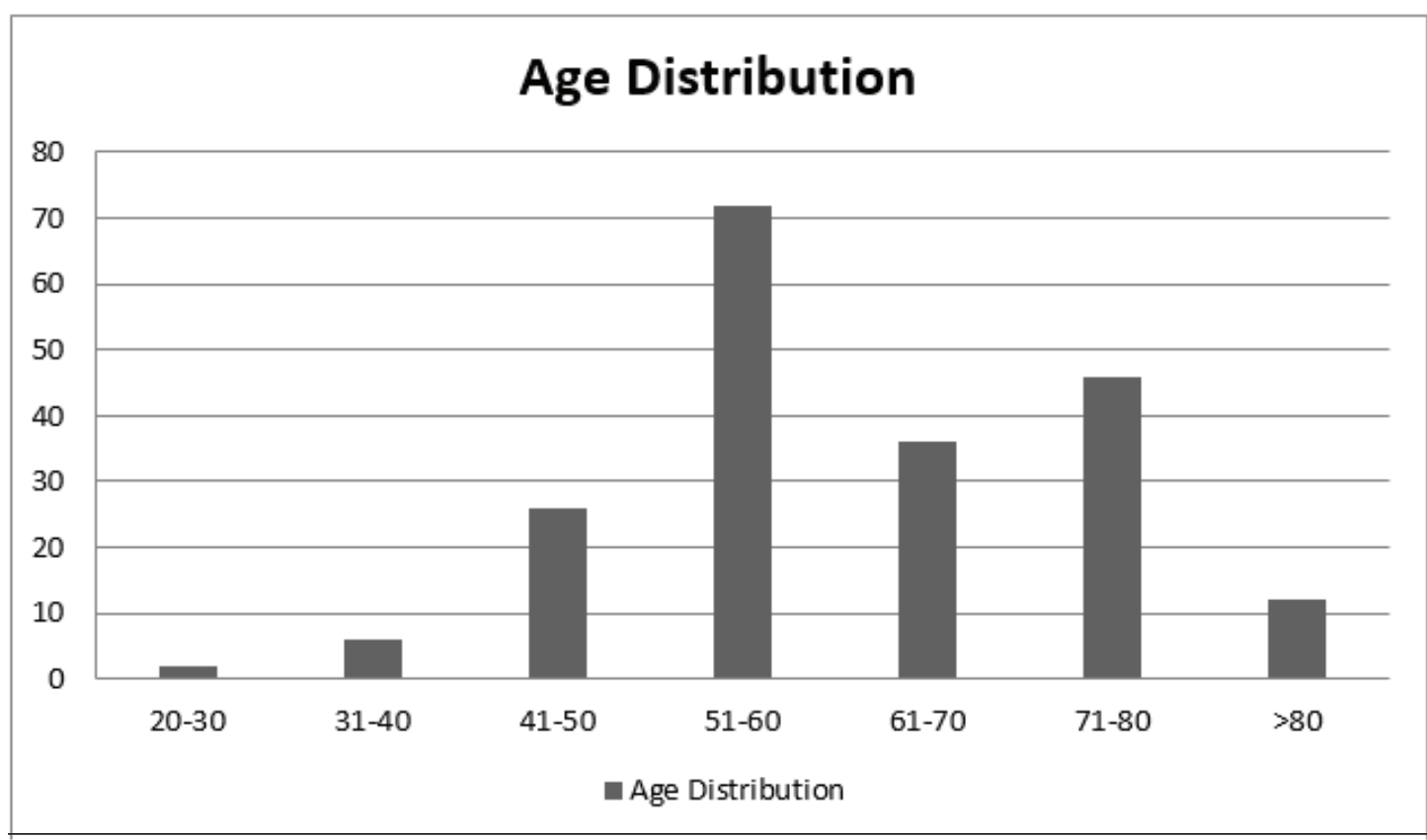

Figure 2: Age distribution among the study population 
8) World Health Organization. Diabetes, Fact sheet [internet]. Nov 2016. Available from :http://www.who.int/mediacentre/factsheets/ fs $312 / \mathrm{en} /$

9) Glycosylated Haemoglobin, HbA1c. Nov 2013. Available from: https:// clinlabnavigator. com/test-interpretations/haemoglobinalc. $\mathrm{html}$ ?letter $=\mathrm{h}$

10) Fowler MJ. Microvascular and macrovascular complications of diabetes. Clin Diabetes. 2008; 26(2):77-82

11) Haffner SM, Lehto $S$, Rönnemaa $T$, Pyörälä K, Laakso M. Mortality from coronary heart disease in subjects with type 2 diabetes and in nondiabetic subjects with and without prior myocardial infarction. $\mathrm{N}$ Engl $\mathrm{J}$ Med. 1998;339(4):229-234.

12) Windler E. What is the consequence of an abnormal lipid profile in patients with type 2 diabetes or the metabolic syndrome? Atheroscler Suppl. 2005;6(3):11-14]

13) American Diabetes Association.Dyslipidemia management in adults with diabetes mellitus. Diabetes Care 2004;24:68-71.

14) The American Association of Clinical Endocrinologists. Medical Guidelines for the Management of Diabetes Mellitus. Jacksonville,FL:AACE;2002. p. 40-82.

15) Taskinen MR. Diabetic dyslipidemia: From basic research to clinical practice. Diabetologia2003;46:733-69.
16) Jamashaid T, Qureshi A. Hyperglyceridemia in Diabetics. Pak Postgrad Med J 2002;13:159-60.

17) Grundy SM. Hypertriglyceridemia, insulin resistance, and the metabolic syndrome. Am J Cardiol2006;83:25-9.

18) American Diabetes Association. Implications of United Kingdom Prospective diabetes study. Diabetes Care 2003;26:28-32.

19) SelvinE,CoreshJ, ShaharE, ZhangL, SteffesM, Sharrett AR Glycaemia (haemoglobinA1c) and incident ischaemic stroke: The Atherosclerosis Risk in Communities (ARIC) Study. Lancet Neurol2005;4:821-6.

20) Haffner SM, LehtoS, RonnemaaT, PyoralaK,LaaksoM.Mortality from coronary heart disease in subjects with Type2 diabetes and in nondiabetic subjects with and without prior myocardial infarction. $\mathrm{N}$ Engl $\mathrm{J}$ Med 1998;339:229-34.

21) Windler $E$. What is the consequence of an abnormal lipid profile in patients with Type 2 diabetes or the metabolic syndrome? Atheroscler Suppl 2005;6:11 4.

22) Mohan V, Shah S, Saboo B. Current glycemic status and diabetes related complications among Type 2 diabetes patients in India: Data from the achieve study. JAPI 2013;61:13-5.

23) Glycosylated Haemoblobin. HBA1C. Available from: hptt://www. clinlabnavigaator.com/testinterpretations/haemoglobin-alc.html? letter=h. [Last updated on 2010 Jun 180]. 